\title{
Condições Crônicas na Atenção Primária à Saúde: Intervenção para Detecção Precoce do Adoecimento Renal
}

\author{
Maria Auxiliadora Resende Sampaio, ${ }^{1}$ Karine da Silva Oliveira, ${ }^{2}$ Juliane Braga da Silva, ${ }^{1}$ \\ Cirliane de Araújo Morais, ${ }^{1}$ Stela Lopes Soares, ${ }^{3}$ Lidia Andrade Lourinho ${ }^{4}$
}

\begin{abstract}
RESUMO
As Doenças Crônicas não transmissíveis são responsáveis por cerca de $63 \%$ do total de óbitos ocorridos no mundo, enquanto no Brasil correspondem a $72,4 \%$ da mortalidade. Assim, a fim de reverter tal cenário, é fundamental realizar não apenas o diagnóstico das condições crônicas de base, mas o seu adequado manejo e, principalmente, a identificação precoce da lesão renal, além do desenvolvimento de ações de prevenção e promoção da saúde como o rastreamento de novos casos da Doença Renal Crônica nas comunidades. O presente estudo teve como objetivo geral desenvolver ações de Educação Permanente (EP) para profissionais de saúde com foco na identificação precoce do adoecimento renal em um Centro de Saúde da Família do município de Sobral/CE. Foi realizada uma pesquisa-intervenção de abordagem qualitativa no mês de dezembro de 2019 em um Centro de Saúde da Família. Participaram da intervenção 24 profissionais e, mediante a ferramenta metodológica do Arco de Maguerez, os participantes julgaram como aplicáveis e de fácil execução a maioria das atribuições e diretrizes norteadoras do cuidado à pessoa com Doença Renal Crônica. Foram elaboradas ações contemplativas a cinco estratégias, com o fim de acompanhamento de pacientes com fatores de risco para Doença Renal Crônica: realização de atividades educativas à população; EPS para os profissionais; estratificação de risco para Hipertensão Arterial Sistêmica, Diabetes Mellitus e Doença Renal Crônica; atendimentos multiprofissionais e melhora da comunicação com a Redes de Atenção à Saúde. É imperativa a continuidade de estudos semelhantes que utilizem da Educação Permanente em Saúde para discutir a Doença Renal Crônica no contexto da Atenção Primária.
\end{abstract}

Palavras-chave: Promoção da saúde. Doença renal crônica. Atenção primária à saúde.

CHRONIC CONDITIONS IN PRIMARY HEALTH CARE: INTERVENTION FOR EARLY DETECTION OF KIDNEY SWEETNESS ABSTRACT

Non-communicable Chronic Diseases account for about $63 \%$ of all deaths worldwide, while in Brazil they account for $72.4 \%$ of mortality. Thus, in order to reverse this scenario, it is essential to carry out not only the diagnosis of chronic underlying conditions, but also their adequate management and especially the early identification of kidney injury, in addition to the development of health prevention and promotion actions such as the screening of new cases of Chronic Kidney Disease in communities. The present study aimed to develop permanent education actions for health professionals with a focus on the early identification of renal illness in a Family Health Center in the city of Sobral/CE. A qualitative intervention research was conducted in December 2019 at a Family Health Center. Twenty-four professionals participated in the intervention and through the methodological tool of arco de Maguerez, the participants judged as applicable and easy to perform most of the attributions and guidelines that guide the care of people with Chronic Kidney Disease. Contemplative actions were elaborated to five strategies with the purpose of monitoring patients with risk factors for Chronic Kidney Disease: The performance of educational activities to the population; EPS for professionals; risk stratification for Systemic Arterial Hypertension, Diabetes Mellitus and Chronic Kidney Disease; multidisciplinary care and improved communication with health care networks. It is imperative to continue similar studies that use Permanent Health Education to discuss Chronic Kidney Disease in the context of Primary Care. Keywords: Health promotion. Chronic kidney disease. Primary health care.

RECEBIDO EM: 22/7/2020

MODIFICAÇÕES SOLICITADAS EM: 20/10/2020

ACEITO EM: 18/11/2020

Escola de Saúde Pública Visconde de Saboia (ESPVS). Sobral/CE, Brasil.

Prefeitura de Sobral. Secretaria Municipal de Saúde de Sobral/CE, Brasil.

Autora correspondente. Centro Universitário Inta - Uninta. R. Antônio Rodrigues Magalhães, 359 - Dom Expedito, 62050-100. Sobral/CE, Brasil. http:// lattes.cnpq.br/6884284520373325. http://orcid.org/0000-0002-5792-4429. stelalopesoares@hotmail.com

4 Universidade Estadual do Ceará (Uece). Fortaleza/CE, Brasil. 


\section{INTRODUÇÃO}

Tradicionalmente, os modos de viver têm sido abordados num contexto individualizante e fragmentário, no qual os indivíduos e as comunidades são posicionados como os únicos responsáveis pelas diversas mudanças/disposições ocorridas no processo saúde-doença ao longo da vida. Sob a ótica ampliada de saúde, como deliberada desde o movimento da Reforma Sanitária, porém, os modos de viver não tratam somente do exercício da vontade e/ou liberdade individual e coletiva, mas, sim, da escolha de determinadas opções de viver como novas possibilidades para satisfazer suas necessidades, desejos e interesses individuais e também que estejam relacionados ao âmbito coletivo (LINO, 2016).

No sistema público de saúde percebe-se o esforço para a garantia dos seus princípios fundamentais e a constante qualificação dos serviços por ele oferecidos e, por melhorar as condições de vida de sujeitos e coletividades por meio da promoção da saúde, compreende-se que é urgente superar a tradição fragmentada e desfocada dos interesses e das necessidades da população (MELO; MESQUITA; MONTEIRO, 2013).

As condições de saúde podem ser definidas como situações na saúde das pessoas que se manifestam de maneira mais ou menos persistentes e demandam respostas sociais reativas ou proativas, episódicas ou permanentes e divididas ou integradas dos sistemas de atenção à saúde, dos profissionais de saúde e dos usuários (MENDES, 2018).

Em 2002 a Organização Mundial de Saúde (OMS) recomendou a implementação da vigilância para doenças crônicas não transmissíveis (DCNTs), com enfoque nos fatores de risco que predizem as mais comuns delas (BASTOS; BREGMAN; KIRSZTAJN, 2017).

O aumento da incidência das condições crônicas é um fato conhecido que tem gerado muitas discussões, compondo, atualmente, um importante problema de saúde pública. Os principais fatores modificáveis de vida nas condições crônicas são os hábitos alimentares inadequados, o sedentarismo e a obesidade, associados às mudanças no estilo de vida, como a cessação do tabagismo, do alcoolismo e o do gerenciamento do estresse psicoemocional (SANTOS et al., 2017).

As DCNTs, das quais faz parte a Doença Renal Crônica (DRC), são responsáveis por cerca de $63 \%$ do total de óbitos ocorridos no mundo, comprometendo milhões de pessoas por ano, enquanto no Brasil, após correções para causas maldefinidas e sub-registro, correspondem a $72,4 \%$ da mortalidade (MALTA et al., 2019).

No desenvolvimento da DRC, alguns fatores de risco já são histórica e cientificamente consagrados, ou seja, há muito tempo são referidos na literatura. Dentre eles podemos destacar a Hipertensão Arterial Sistêmica (HAS), por ocasionar lesão no capilar glomerular, o Diabetes Mellitus (DM), uma vez que os altos níveis de glicemia, de maneira continuada, podem levar a alterações hemodinâmicas e funcionais nos glomérulos, o histórico familiar, uma vez que os familiares de pacientes com DRC apresentam prevalência aumentada de doença renal, e, por fim, os fatores relacionados ao estilo de vida do paciente, como o tabagismo e o sedentarismo (ABRAHÃO, 2016).

Os pacientes com DRC apresentam um aumento na sobrevida em razão do emprego da terapia renal de substituição. Apesar disto, estudos demonstraram o impacto negativo que a doença e o tratamento desencadeiam nos pacientes sobre os seus sistemas orgânicos, o que interfere na saúde física e mental, na sua funcionalidade, independência, bem-estar geral e no convívio social. Isto repercute na redução da capacidade funcional e força muscular dos pacientes (FASSBINDER et al., 2015).

Assim, podemos constatar a magnitude dessa morbidade, cuja incidência aumenta cada vez mais de forma alarmante e tem como principal etiologia complicações advindas de condições como hipertensão arterial e diabetes mellitus não controlados ou manejados inadequadamente.

A fim de reverter tal cenário, é de fundamental importância não apenas o diagnóstico das condições crônicas de base (como HAS e DM), mas o seu adequado manejo e, principalmente, a identificação precoce da lesão renal (ainda em suas fases iniciais) por meio do seu estadiamento, além do desenvolvimento de ações de prevenção e promoção da saúde, com o controle rigoroso de seus níveis pressóricos, glicêmicos, lipídicos, suspensão do tabagismo, além da referência para o especialista em tempo hábil e do rastreamento de novos casos desta doença nas comunidades (CALDAS et al., 2013).

O profissional enfermeiro que atua como promotor da saúde deve respeitar as experiências do paciente e conduzir de maneira adequada cada caso. É de sua competência, enquanto integrante da equipe multiprofissional, o desenvolvimento de atividades de promoção da saúde individuais e coletivas que per- 
meiem as condições e características de saúde modificáveis no intuito de prevenir as morbidades ou controlá-las (PEREIRA et al., 2013).

A enfermagem atua com papel fundamental na assistência ao paciente, mas também como ciência promotora de saúde. Compreende-se que o enfermeiro deve intervir junto aos pacientes, incentivando-os a uma alimentação saudável, prática regular de exercício físico adequado assim como o controle de determinadas condições que podem já estar alteradas, como a hipertensão, a taxa glicêmica e as dislipidemias (DIAS, 2014).

Justifica-se a escolha da temática a partir da análise dos dados do último levantamento realizado em novembro de 2018 no Centro de Saúde da Família, que será cenário para a intervenção. As informações apontam para os preocupantes índices de pessoas usuárias acometidas com as doenças crônicas aqui discutidas (391 pessoas com hipertensão arterial e 167 com diabetes mellitus), o que demanda com urgência o olhar para a identificação do adoecimento renal neste público.

Ainda justificando a presente abordagem, considera-se a vivência de uma das pesquisadoras, enquanto especialista na enfermagem em nefrologia, e também como promotora da saúde no nível primário de atenção, quando a enfermagem, enquanto ciência holística, é capaz de atuar ante os diversos desafios enfrentados pelo paciente em adoecimento renal.

A partir do panorama exposto, compreende-se que há expressivos índices sobre os benefícios na qualidade de vida do paciente, provenientes do acompanhamento adequado bem como do rastreio precoce da lesão renal pela Atenção Primária à Saúde (APS). Buscou-se, dessa forma, o aprimoramento deste acompanhamento por meio do conhecimento de estratégias e intervenções adequadas de assistência à saúde de pessoas com DRC.

Compreende-se a relevância de estudos com esta proposta, tendo em vista a complexidade do cuidado aos pacientes com fatores de risco ou DRC já instalada. $\mathrm{O}$ Estudo pretendeu fomentar a prática profissional de modo a qualificar o cuidado prestado ao paciente, que, por sua vez, terá maior qualidade de vida e menor risco de complicações advindas do deficiente controle de condições crônicas.

Para fundamentar o presente estudo, tem-se como objetivo desenvolver ações de Educação Permanente para profissionais de saúde com foco na identificação precoce do adoecimento renal em um Centro de Saúde da Família (CSF) do município de Sobral/CE.

\section{MÉTODO}

O estudo constituiu-se de uma pesquisa-intervenção de abordagem qualitativa acerca de um plano de intervenção com foco na detecção do adoecimento renal pela Atenção Primária à Saúde.

Para Mendes, Pezzato e Sacardo (2016), a pesquisa-intervenção propõe uma intervenção de ordem micropolítica na experiência social e trata de ampliar o escopo teórico-metodológico das pesquisas participativas. Dessa forma, as caracteristicas da pesquisa-intervenção vêm proporcionando o desenvolvimento de espaços de problematização coletiva junto as práticas de formação e potencializando a produção de um novo pensar/fazereducação.

A intervenção foi realizada no mês de dezembro de 2019 no Centro de Saúde da Família (CSF) Cleide Cavalcante Sales, no município de Sobral-CE.

Sobral é uma cidade localizada na zona norte do Estado do Ceará, a uma distância de 238 quilômetros de Fortaleza e possui uma população estimada em aproximadamente 203.682 habitantes (IBGE, 2016). O município tem sua Atenção à Saúde organizada por níveis de atenção, sendo referência para 55 municípios da zona norte do Estado do Ceará. Possui um Sistema de Saúde Escola que é referência nacional para a Atenção Primária à Saúde, e atualmente conta com 63 Equipes de Saúde da Família (eSF), atuando em 35 CSF, onde 20 estão localizados na sede e 15 na zona rural do município (SOBRAL, 2016).

O CSF está situado no Bairro Sumaré, e sua área de abrangência compreende um total de 6.579 pessoas, agrupadas em um 1.946 famílias, correspondendo a um número médio de 3,42 pessoas por família. Possui duas Equipes de Saúde da Família (ESF) e duas Equipes de Saúde Bucal (ESB), bem como duas equipes de apoio: Núcleo Ampliado de Saúde da Família e Atenção Básica (Nasf-AB) e Residência Multiprofissional em Saúde da Família (RMSF), ambas contemplando profissionais das seguintes categorias: educação física, nutrição, psicologia, fisioterapia, enfermagem e serviço social (SOBRAL, 2016).

Como referido anteriormente, os dados do último levantamento realizado em novembro de 2018 informam que, em se tratando das doenças crônicas aqui discutidas, têm-se o número total de 391 pessoas acometidas por hipertensão arterial e 167 por diabetes mellitus somente no território do CSF Sumaré (SOBRAL, 2016).

Participaram da intervenção os profissionais da Equipe de Saúde da Família/Saúde Bucal (1 médica, 1 enfermeira, 2 técnicos de enfermagem, 10 agentes co- 
munitários de saúde e 2 dentistas) e das equipes multidisciplinares de apoio, Nasf-AB e RMSF (2 profissionais de educação física, 1 fisioterapeuta, 2 assistentes sociais, 1 psicóloga e 2 nutricionistas).

A amostra dos profissionais participantes foi formada a partir dos seguintes critérios de inclusão: profissional atuante na Unidade Básica de Saúde (UBS) que constituiu o cenário do estudo e que estava regulamente cadastrado no Sistema de Cadastro $\mathrm{Na}$ cional de Estabelecimentos de Saúde (SCNES). Foram excluídos aqueles profissionais que estavam em período de férias, com atestado médico ou licença durante a intervenção, ou, ainda, aqueles que não puderam se ausentar de suas atividades nos momentos da intervenção.

A busca pela compreensão do objeto do estudo na perspectiva da pesquisa enquanto ferramenta de construção do conhecimento e transformadora da realidade, levou-nos a adaptar as etapas do método do Arco de Maguerez para subsidiar os pressupostos da intervenção participativa.

O método trata de uma estratégia de ensino-aprendizagem para o desenvolvimento da Problematização. Desenvolve-se em cinco etapas que acontecem a partir da realidade social: a observação da realidade, os pontos-chave, a teorização, as hipóteses de solução e aplicação à realidade (BORDENAVE; PEREIRA, 2004).

No primeiro momento foram apresentados os objetivos e etapas do projeto de intervenção a todos os profissionais do CSF na ocasião da reunião mensal da equipe, a fim de que conhecessem a intervenção que aconteceria na Unidade bem como todos os profissionais que estariam envolvidos.

O Termo de Consentimento Livre e Esclarecido (TCLE) foi apresentado e explanado a todos os profissionais participantes da intervenção, que, logo após, foram convidados a assiná-lo.

Considerando os meios para o alcance das metas traçadas neste projeto, as etapas foram desenvolvidas por intermédio da relação entre os objetivos da intervenção e as etapas do Arco de Charles Maguerez, as quais estão descritas a seguir.

\section{Primeira etapa: Observação da Realidade}

As fases descritas foram desenvolvidas nos encontros de Educação Permanente propostos para o alcance dos objetivos traçados neste estudo. A temática geral foi introduzida por meio da observação crítica da realidade estudada, conforme orienta o método em questão.
Para Villardi, Cyrino e Berbel (2015), esta etapa gera o levantamento inicial das informações da realidade a fim de problematizá-la a partir dos saberes e experiências que traz consigo.

Desta forma, no momento inicial de observação da realidade pretendeu-se elaborar justificativas para a escolha do problema bem como elucidar as contribuições para o meio. Esperou-se, com esta etapa, o envolvimento intelectual e político dos profissionais com a realidade exposta.

Mediante exposição dialogada, foram citados os principais indicadores relacionados à hipertensão arterial e à diabetes mellitus como fatores de risco para a Doença Renal Crônica bem como os dados que expressam o seu contexto na realidade (taxas de pessoas com condições crônicas que evoluem para DRC, efeitos da DRC na qualidade de vida do usuário, custos da terapia renal substitutiva). As opiniões geradas pela discussão foram registradas conforme orienta a estratégia do diário de campo, a qual será mais bem descrita na etapa avaliativa da intervenção.

É o momento em que os sujeitos envolvidos podem olhar atentamente para a realidade, escolhendo aspectos que precisem ser desenvolvidos, trabaIhados, revisados ou melhorados (VILLARDI; CYRINO; BERBEL, 2015).

\section{Segunda etapa: Determinação de pontos-chave}

Borba et al. (2015) afirmam que, de acordo com o arco de Maguerez, este é o momento para ser definido e discutido o aspecto do problema que será objeto de pesquisa.

Após a discussão inicial foi promovida uma reflexão ou questionamento sobre os possíveis fatores associados ao desenvolvimento da DRC e que são determinantes na sua existência. Depois dessa reflexão inicial, os profissionais foram estimulados à discussão sobre os possíveis determinantes maiores, contextuais, tais como os aspectos políticos e econômicos, que podem estar associados ao problema e aos próprios fatores járegistrados.

Para Klein e Barin (2018), nas discussões os sujeitos vão estabelecendo os aspectos essenciais para compreender mais densamente o problema e encontrar caminhos de intervir na realidade. Nessa etapa de análise reflexiva, o facilitador orientou que os participantes elaborassem uma síntese, apontando o conjunto dos tópicos a serem investigados.

Desta forma, após a discussão foram descartados os fatores superficiais e contingentes da discussão, e elencados e registrados em papel madeira e em 
diário de campo os pontos-chave reconhecidos como problemas fundamentais que interferem no rastreio da doença renal pela APS.

\section{Terceira etapa: Teorização}

No segundo encontro de EP, após definidos os pontos-chave da problematização, foi iniciada a fase da teorização que, conforme Villardi, Cyrino e Berbel (2015), trata da busca do conhecimento e de dados acerca do problema em variadas fontes, que servem de base para a transformação da realidade.

Os profissionais foram instrumentalizados com o protocolo de diretrizes clínicas para o cuidado ao paciente com DRC no SUS do Ministério da Saúde (2014), e, por meio de exposição dialogada, puderam fomentar as discussões estabelecidas na fase anterior bem como questionar a aplicabilidade das diretrizes no campo de atuação da APS e realidade territorial local.

Foram levantadas as principais contribuições teóricas acerca da fisiopatologia renal, manejo da HAS, DM e demais fatores de risco com vistas ao retardo da progressão da lesão renal, ferramentas e dispositivos de diagnóstico e estadiamento da lesão renal e sua aplicabilidade na realidade local, bem como opções terapêuticas: noções sobre o tratamento conservador e Terapia Renal Substitutiva (TRS).

A exposição dialogada como metodologia ativa da EP permitiu o envolvimento de todos os profissionais na discussão, o que foi de grande importância, posto que o momento não se resumiu em aquisição de novas informações aos profissionais, mas na sua sensibilização para a problemática em seu cotidiano de trabalho.

É importante ressaltar que tal fundamentação teórica serviu como embasamento para os profissionais que, na etapa seguinte, discutiram as primeiras hipóteses para solução da problemática.

\section{Quarta etapa: Hipóteses de solução}

Ainda no segundo encontro da EP foram discutidas as principais ideias a serem trabalhadas na prática para minorar a problemática em discussão. A quarta etapa de problematização do arco de Maguerez orienta a discussão supracitada e propõe que o potencial criativo-reflexivo seja mobilizado para o participante pensar de modo inovador (KLEIN; BARIN, 2018)

As autoras sugerem, ainda, algumas questões que podem ser feitas nessa etapa com o intuito de instigar os participantes à reflexão daquilo que será apontado como possível solução: O que é necessário para chegar a uma solução para o problema? O que deve ser providenciado? O que pode ser feito de fato?

Todos os participantes foram instigados e estimulados a apresentar contribuições para minorar a problemática em discussão.

A contribuição da autora para esta fase foi a exposição de um instrumento norteador para a investigação e o acompanhamento dos usuários com fatores de risco ou DRC já instalada. O documento contém questões sobre a patologia de base, comorbidades e outros fatores de risco, exames realizados, campo para cálculo da taxa de filtração glomerular (TFG) e estadiamento da lesão renal.

Nesta ocasião foi discutida a aplicabilidade do referido instrumento e seus objetivos, bem como sobre o preenchimento adequado de cada item, com as devidas orientações para a sua utilização por parte dos profissionais.

\section{Quinta etapa: aplicação prática à realidade}

A quinta fase proposta pela metodologia aqui discutida versou sobre a aplicação das propostas mais viáveis de solução do problema no todo ou em parte, o que contribuiu na transformação da realidade investigada (VILLARDI; CYRINO; BERBEL, 2015).

Neste terceiro e último encontro de EP os profissionais utilizaram o instrumento apresentado na etapa anterior com casos fictícios. Os profissionais foram divididos em pequenos grupos para a resolução dos casos com a utilização do instrumento.

Neste momento foram sanadas as dúvidas que surgiram a respeito da ferramenta em questão, bem como de outras estratégias propostas.

A avaliação em saúde requer um investimento cuidadoso e consistente na construção de uma série de consensos. Trata-se não apenas da necessidade de compatibilização de instrumentos, mas de se pactuar o objeto e os objetivos da avaliação, compreendendo-se que esta é, antes de tudo, uma atividade negociada entre diferentes atores do sistema de saúde. Sem isso, torna-se inviável realizar qualquer ação que esteja comprometida com uma maior equidade e efetividade dos serviços de saúde (BRASIL, 2014).

Para a execução da avaliação é necessário serem respeitados alguns dos principais elementos do processo avaliativo, que são: os objetos da avaliação, momentos, critérios, a função da avaliação, os atores da avaliação e o desenho metodológico (SERAPIONI, 2012). 
A educação permanente foi avaliada por meio de questionário aplicado individualmente, contendo questões para uma melhor compreensão dos possíveis impactos destes momentos no processo de trabalho dos profissionais e também para avaliar o grau de satisfação dos mesmos. A grande vantagem é sua facilidade de manuseio, pois é fácil a um pesquisado emitir um grau de concordância sobre uma afirmação qualquer, contribuindo positivamente para sua aplicação nas mais diversas pesquisas (COSTA, 2011).

$A$ avaliação da EP, do instrumento proposto, bem como de outras estratégias de solução, foi realizada ao final do terceiro e último momento.

Os profissionais foram convidados a formar uma roda de conversa para a discussão e foram consideradas as suas contribuições para a utilização do instrumento, o qual foi disponibilizado para uso pela equipe e poderá ser empregado em futuras pesquisas com casos reais.

A perspectiva do diário de campo tem se mostrado amplamente potente nas pesquisas em saúde. O caderno de notas é usado pelo pesquisador para registrar as conversas informais, observações do comportamento durante as discussões, manifestações dos interlocutores quanto aos pontos investigados e ainda suas impressões pessoais, que podem modificar-se com o decorrer do tempo (ARAÚJO et al., 2013).

A intervenção seguiu os protocolos éticos para pesquisa com seres humanos conforme as exigências éticas e científicas fundamentais descritas pela Resolução 466/12 de dezembro de 2012 do Conselho Nacional de Saúde (CNS), as quais discorrem acerca das Diretrizes e Normas Regulamentadoras de pesquisas envolvendo seres humanos, e também foi submetida à Comissão Científica da Secretaria de Saúde de Sobral, que emitiu parecer favorável no 0257/2018 e, posteriormente, ao Comitê de Ética em Pesquisa (CEP) por meio da plataforma Brasil.

Destaca-se que foram respeitados os princípios éticos da pesquisa, garantindo a veracidade dos resultados, a minimização dos riscos e o sigilo das identidades, evitando-se a possibilidade de contrangimento do participante. Desta forma, respeitamos os princípios da beneficência, havendo discernimento entre riscos e benefícios atuais e potenciais, de forma que nos comprometemos com o máximo de benefícios e o mínimo de riscos e danos; da não maleficência, quando garantimos que danos previsíveis seriam evitados; da autonomia, pela qual os participantes tiveram total liberdade de desistir de participar do estudo em qualquer etapa do processo se assim achassem necessário, sem que isso lhes trouxesse algum dano ou prejuízo; e o princípio da justiça e equidade, quando justificamos a realização do estudo em sua relevância social (BRASIL, 2012).

O Termo de Consentimento Livre e Esclarecido (TCLE) foi disponibilizado aos participantes para leitura, discussão e posterior assinatura após o esclarecimento dos objetivos da presente intervenção.

\section{RESULTADOS E DISCUSSÕES}

A metodologia utilizada para o alcance dos objetivos permitiu a discussão da temática de maneira ampla. A proposta do Arco de Maguerez favoreceu a aproximação da equipe à temática. De maneira gradativa, os participantes familiarizavam-se com o assunto e suas características.

\section{Aproximando a realidade problematizada}

A primeira etapa proposta permitiu que os participantes iniciassem uma reflexão em torno da problemática mediante a exposição dos principais indicadores de saúde que envolvem a Doença Renal Crônica na atualidade.

Estudos de gráficos, números e censos permitiram aos sujeitos a visualização da realidade da DRC em âmbito mundial, nacional e até mesmo municipal. Observar a problemática e compreender que esta também contempla aquele território, despertou o interesse dos participantes que, a todo o momento, manifestavam-se por meio de falas e questionamentos, como é possível perceber a partir dos registros em diário de campo:

É interessante porque a gente costuma pensar que este é um problema longe da nossa realidade e, na verdade, nós é que não conseguimos enxergar o problema como ele é (Participante 1 - Agente Comunitário de Saúde).

A sequência de gráficos que exemplificavam as principais causas da DRC e mostravam a preponderância da Hipertensão Arterial Sistêmica e Diabetes Mellitus bem como a importância da Atenção Primária à Saúde, causaram importante impacto aos sujeitos participantes, os quais socializaram a densidade de pacientes do território com as referidas patologias, afirmando, assim, a necessidade de se discutir aquele tema.

Desde a primeira etapa do método algumas colocações dos participantes demonstravam a sua curiosidade no âmbito do fazer profissional daquela equipe para reverter ou minorar a situação de saúde pública apresentada, o que já demonstrou entendimento do problema, pois, conforme afirmam Hossain e Laditka (2009), há impacto positivo do trabalho da APS nos desfechos de condições de saúde. 


\section{Decifrando os aspectos fundamentais}

A segunda etapa do Arco de Maguerez permitiu o diálogo em torno dos fatores associados ao desenvolvimento da DRC, seus determinantes maiores e aqueles cuja governabilidade é maior por parte da equipe de saúde.

A discussão foi endossada por quase todos os presentes que manifestavam diferentes condições de trabalho vivenciadas por eles.

As colocações versaram desde a estrutura física da unidade, a concessão dos exames laboratoriais, os encaminhamentos ao plano secundário, a fragilidade da comunicação entre as Redes de Atenção e, até mesmo, o (sub)financiamento do SUS, que refletia diretamente em todas aquelas problemáticas e na assistência prestada à comunidade. Os profissionais reconheceram este como o principal desafio a ser superado, corroborando a ideia de Reis et al. (2016), que afirmam que assegurar um financiamento público adequado que viabilize a garantia do direito à saúde prevista na Constituição Federal de 1988, tem sido um dos maiores desafios para o SUS.

Após as discussões e análises reflexivas, o grupo de participantes foi orientado a elaborar uma síntese e apresentar o conjunto de tópicos apontados como pontos-chave a serem considerados nas demais etapas da EP, e, logo, foram definidos alguns problemas fundamentais para o rastreio da doença renal pela APS:

- Demora nos resultados de exames laboratoriais;

- Falta de conhecimento do paciente acerca das possíveis complicações de doenças de base;

- Falta de discussão sobre a DRC na APS;

- Fragilidade na garantia dos espaços de Educação Permanente;

- Desconhecimento do paciente sobre os fluxos;

- Profissionais insuficientes para garantir a qualidade do cuidado;

- Dificuldades na realização/atualização de estratificações de risco de HAS e DM.

\section{Buscando o conhecimento evidenciado}

O segundo encontro de EP teve início a partir de uma síntese acerca das duas etapas discutidas no momento anterior. Foram expostos os pontos-chave antes elencados, de modo que o participante pudesse consultá-los a cada reflexão.
Para Prado et al. (2012), a teorização como etapa da metodologia do Arco permite aos sujeitos a percepção do problema e o questionamento dos acontecimentos observados nas fases anteriores. Consoante os autores, uma teorização bem desenvolvida leva o sujeito a entender as questões não somente em suas manifestações baseadas nas experiências ou situações, mas também as contribuições teóricas que os explicam. Nesse momento de teorização acontecem as ligações mentais de análise que favorecem o amadurecimento intelectual.

Desta forma, os participantes foram instrumentalizados com a Portaria no 1.675 de 7 de junho de 2018, que dispõe sobre os critérios para a organização, funcionamento e financiamento do cuidado da pessoa com Doença Renal Crônica no âmbito do Sistema Único de Saúde, bem como com as Diretrizes Clínicas para o cuidado ao paciente com DRC no SUS. A equipe conheceu os documentos norteadores e debruçou-se sobre as competências do componente da Atenção Primária.

De acordo com o protocolo de diretrizes, o cuidado com o paciente com DRC no SUS está organizado em razão do estadiamento da doença segundo o cálculo da Taxa de Filtração Glomerular (TFG) (BRASIL, 2014). Assim, foi iniciada a discussão sobre a classificação da DRC em estágios e a maneira de se classificar cada paciente por meio do cálculo da sua TFG. Os participantes conheceram as principais fórmulas para o cálculo, bem como o aplicativo de celular desenvolvido pela Universidade Federal do Rio Grande do Sul e os sites que calculam a referida taxa mediante preenchimento dos dados do paciente.

Após a compreensão teórica da estratificação, passo inicial para organizar o cuidado com a pessoa com DRC, os participantes mostraram-se entusiasmados com a ferramenta prática e necessária que acabavam de conhecer, como é possível observar nas falas registradas em diário de campo:

Com a prática da estratificação a gente vai poder conhecer melhor as pessoas que têm fatores de risco para DRC ou até mesmo aquelas que já têm algum grau da doença (Participante 2 - Médica).

A estratificação é o primeiro passo de tudo. Agora vamos poder organizar melhor os atendimentos em nível de atenção semelhante (Participante 3 - Enfermeira).

A colocação supracitada das profissionais vai ao encontro do já explanado por Silva (2018), quando a autora afirma que os aspectos relacionados à estratificação de risco merecem atenção, pois evitam que 
se empreendam esforços e recursos em intervenções desnecessárias, equivocadas e ineficientes, como também aumenta o risco de essas pessoas progredirem para um quadro de complicações.

Os participantes ainda relataram a facilidade na utilização do aplicativo para calcular a TFG dos pacientes e assim estratificar o estágio ao qual pertencem:

Agora a gente entende como estratificar e qualquer pessoa que está aqui pode fazer, desde que tenha entendido bem (Participante 1 - Agente de Saúde).

\section{Inovando em Soluções Práticas}

Em seguida, e já como introdução à fase de elaboração de hipóteses de solução, os participantes foram divididos em duplas e fez-se a leitura detalhada da portaria no 1.675/18, em que discutiram a aplicabilidade de cada competência descrita, sendo orientados a refletirem sobre as possíveis estratégias para melhorar/garantir a execução das principais atribuições.

Foram convidados, após conversa em duplas, para a socialização das discussões no grupo maior. Neste momento tomou-se nota das contribuições colocadas pelos participante.

Percebeu-se, com a leitura e discussão, que muitas ações já deveriam estar implícitas no trabalho da equipe de APS, por exemplo, a promoção da saúde voltada ao controle de doenças como hipertensão e diabetes. Os participantes propuseram, então, estratégias para fortalecer suas práticas diárias e adotarem novas práticas também necessárias.

Realizadas as primeiras discussões, os participantes foram instrumentalizados com a tabela $5 \mathrm{~W} 2 \mathrm{H}$ adaptada para que pudessem traçar, a partir das estratégias preestabelecidas, ações práticas a serem desenvolvidas pela equipe.

De acordo com Avila et al. (2016), a tabela $5 \mathrm{~W} 2 \mathrm{H}$ consiste em um plano de ação para atividades preestabelecidas que necessitam ser executadas com a maior clareza possível, e traça um desenho das mesmas por meio do objetivo principal da ferramenta, que é responder a algumas questões básicas e organizá-las.

No caso desta intervenção, a tabela foi adaptada e procurou responder a três questões previstas: $\mathrm{O}$ quê? Como? e Quando? O objetivo primordial neste momento era que os participantes descrevessem ações palpáveis aos participantes já com o período previsto de início e frequência de realização. Assim, foram traçadas ações a partir de cinco eixos: Atividades educativas à população; EPS para os profissionais; Estratificação de risco para HAS, DM e DRC; Atendimentos multiprofissionais; e Melhora da comunicação com a RAS.

Como é possível observar, com a utilização da ferramenta $5 \mathrm{~W} 2 \mathrm{H}$ as ações traçadas tornaram-se palpáveis e possíveis de executar. A tabela foi disponibilizada para cada equipe para a realização do planejamento e adequação dos seus cronogramas e agendas.

Tendo em vista as categorias de ações propostas, é possível reconhecer a necessidade da realização de atividades de educação em saúde, as quais são recomendadas desde a Política Nacional de Atenção Básica (PNAB), que destaca as atribuições dos profissionais da equipe da Estratégia Saúde da Família, orientando que devem realizar tais ações à população adstrita com planejamento em equipe e utilizando abordagens adequadas às necessidades deste público (BRASL, 2017).

Do mesmo modo, a Política Nacional de Educação Permanente vem refletir acerca da aprendizagem significativa e da possibilidade de transformar as práticas profissionais, o que a configura como uma aba educacional importante e contribui com a reflexão sobre o processo de trabalho mediante a proposta do aprender a aprender (BRASIL, 2017).

A discussão permeou ainda o contexto da estratificação de risco das condições crônicas mais prevalentes no território, como a HAS e DM, bem como da DRC, posto que a equipe passou a conhecê-la a partir da presente intervenção aqui relatada. As colocações versaram acerca da necessidade da estratificação para o adequado manejo da condição do paciente.

Por outro lado, a equipe relata dificuldade na estratificação de $100 \%$ da população, bem como em mantê-las atualizadas, o que interfere diretamente no cuidado prestado, corroborando o afirmado por Silva (2018), que relata serem insuficientes as práticas clínicas relacionadas a condições crônicas, como a HAS e DM, principalmente quando se trata da introdução de ações conforme a estratificação de risco da população assistida, tornando-se empecilho para o alcance de melhores resultados na assistência baseada no conhecimento científico recomendado.

Ainda como recomendação para a prevenção da DRC a partir do cuidado à população com doença crônica, os participantes abordaram o atendimento multiprofissional ofertado na unidade. Propuseram ações de modo que favorecessem o atendimento integral ao paciente, o que lembra Mendes (2012), que 
afirma que, desta forma, os usuários são beneficiados em ganhos de tempo e de conforto, na facilidade de se relacionar, num mesmo dia, com diferentes áreas do saber, em consultas programadas em sequência ou em grupos de profissionais.

A intervenção permitiu ainda a discussão em torno da posição da APS na Rede de Atenção à Saúde (RAS) e a importância de se efetivar a comunicação entre os seus diversos pontos.

Para Mendes (2012), a relação de parceria entre os especialistas e os médicos de APS deve se pautar numa comunicação fluida. No modelo mais comum de referência e contrarreferência, o médico de APS deve consultar o especialista especialmente em situações como: dificuldade em fazer um diagnóstico, metas de tratamento não atingidas e tratamento muito especializado.

\section{Praticando as Soluções Inovadoras}

Como propõe a metodologia utilizada, na fase de elaboração de hipóteses de solução o facilitador do momento deve contribuir com os participantes apresentando alguma estratégia a ser utilizada e aplicada à realidade para a solução do problema. Desta forma, foi apresentado ao coletivo o instrumento norteador para a investigação e o acompanhamento dos usuários com fatores de risco ou DRC já instalada. Foi realizada leitura coletiva do documento, esclarecendo cada item contido nele e com a devida orientação para o preenchimento.

Os participantes receberam uma cópia do referido documento e a utilizaram em um caso clínico fictício apresentado. $O$ instrumento permite o conhecimento de informações de saúde do paciente, bem como a estratificação segundo o grau de risco da pessoa já acometida por algum grau de lesão renal.

\section{Avaliando o produto}

Finalizadas as etapas do Arco, os participantes foram convidados ao momento de avaliação do instrumento e da EP realizada. Por intermédio da roda de conversa, facultou-se a fala para aqueles que desejassem contribuir com a fase avaliativa referente ao instrumento proposto. A equipe foi orientada a acrescentar o seu ponto e vista acerca da ferramenta e suas contribuições para modificação e uso da mesma. Os registros de falas do diário de campo permitiram compreender a visão dos participantes após o momento:

O instrumento é bem fácil de usar e a estratificação parece bem mais simples que a de HAS e DM (Participante 4 - Nutricionista).
Realmente o caso apresentado é bem comum de acontecer na nossa realidade e com o instrumento e a estratificação já poderemos agir e evitar complicações para o paciente (Participante 3 Enfermeira).

O instrumento pede informações fundamentais para conhecermos o paciente e todas de fácil acesso (Participante 5 - Agente de Saúde).

É um excelente norteador que podemos usar nas consultas diante de um paciente hipertenso, diabético ou com outros fatores de risco pra DRC, e seria muito interessante que se realizasse uma pesquisa de campo que avaliasse o seu uso na prática clínica (Participante 2 - Médica).

O instrumental de avaliação da EP continha ainda uma questão subjetiva para que os participantes pudessem descrever, em síntese, de que maneira a EP poderia interferir na sua prática profissional. A seguir estão transcritas algumas das principais colocações:

As doenças crônicas não transmissíveis são de grande importância para a saúde pública e atualmente trabalhamos muito com a hipertensão e diabetes, mas a doença renal ficava esquecida e, agora, após a EP, olharei para os pacientes com uma visão mais qualificada.

A EP me trouxe novos conhecimentos sobre o assunto, me ajudando a crescer profissionalmente e melhorando minha postura na comunidade.

A EP valorizou a interdisciplinaridade no cuidado à saúde, sendo de importante impacto para a minha prática e, assim, interferiu positivamente.

Me ajudou a nortear como identificar o paciente com DRC e a cuidar desse paciente acompanhando seu histórico, muito produtivo.

Abriu-me os olhos sobre prevenção e diagnóstico precoce da DCR. Chamou-me atenção para possíveis casos de DRC no território.

Serviu-me para ter mais atenção no atendimento ao paciente com DM e HAS visto o seu maior risco de DRC, momento rico que nunca tinha sido abordado como EP.

Serviu para termos mais atenção também às queixas do paciente.

Principalmente no conhecimento do aplicativo e do instrumento, facilitando a avaliação do paciente com rapidez.

Compreende-se, portanto, a importância do aprimoramento profissional tendo em vista que na educação permanente o processo educativo põe em análise 
o cotidiano do trabalho ou da formação em saúde. Ela constitui, portanto, uma estratégia que possibilita uma formação realmente sintonizada com as necessidades da saúde pública brasileira (VIANA et al., 2015).

\section{CONSIDERAÇÕES FINAIS}

O foco do presente estudo sobre intervenção desenvolveu momentos de Educação Permanente em Saúde para profissionais de uma Unidade Básica de Saúde e interviu diretamente na qualidade do atendimento prestado à população, principalmente à parcela de usuários com condições crônicas, como hipertensão e diabetes, prevalentes patologias no território em questão.

Mediante a participação ativa e o relato dos participantes, é possível compreender a necessidade da abordagem da Doença Renal Crônica no âmbito da Atenção Primária à Saúde. Tal necessidade foi relatada por diferentes categorias, mesmo as mais próximas da prática clínica.

A ferramenta do Arco de Maguerez mostrou-se essencial para a abordagem da temática, pois permitiu problematizar a DRC desde seus aspectos epidemiológicos, o que contribuiu para a que a equipe compreendesse a dimensão da questão e a parcela de atribuições deste nível de atenção. Possibilitou, ainda, a compreensão de pontos fundamentais do problema e a elaboração de hipóteses para minorar ou solucionar a questão, além de aplicá-las à realidade do território.

A teorização instrumentalizada pelas diretrizes preconizadas para a prevenção da DRC pela equipe da Estratégia Saúde da Família, permitiu a compreensão de diversas práticas profissionais já intrínsecas ao trabalho da APS, como a promoção da saúde relacionada às doenças crônicas e a estratificação segundo o grau de risco de hipertensão e diabetes. Os participantes julgaram como aplicáveis e de fácil execução a maioria das atribuições e diretrizes norteadoras do cuidado, com exceção daquelas cuja governabilidade não está sobre a equipe, como é o caso das cotas para realização de exames laboratoriais, a marcação de consultas especializadas em tempo oportuno e o (sub)financiamento do sistema Único de Saúde, fatores que interferem diretamente na assistência prestada.

A partir do conteúdo das discussões foram elaboradas, também, ações contemplativas a cinco estratégias, com o fim de acompanhamento de pacientes com fatores de risco para DRC, como a realização de atividades educativas à população; EPS para os profissionais; estratificação de risco para HAS, DM e DRC; atendimentos multiprofissionais e melhora da comunicação com a RAS.
$\mathrm{O}$ instrumento norteador proposto gerou contribuições positivas à intervenção, que recomendou a sua utilização em futuros estudos de campo bem como na prática clínica, posto que o mesmo norteia e facilita o processo de estratificação de risco das pessoas com Doença Renal Crônica e viabiliza o melhor acompanhamento destas.

Ao analisar os resultados da avaliação realizada pode-se compreender que a EP teve sua importância reconhecida pelos participantes que a julgaram como temática atual em saúde pública, de compreensão fácil, linguagem interativa, informações objetivas, incentivando-os a aprimorarem suas condutas profissionais e fortalecendo sua aprendizagem.

A partir das contribuições sobre promoção da saúde realizadas e promovidas pela intervenção, compreendemos que no contexto da integração educação-saúde a transformação das práticas demanda um empenho coletivo e consciente para superar os desafios e desenvolver conveniências para a qualificação da atenção à saúde e para a melhoria destas ações.

É imperativa a continuidade de estudos semeIhantes que utilizem a Educação Permanente em Saúde para discutir a Doença Renal Crônica no contexto da Atenção Primária, notadas as contribuições positivas ao paciente com fatores de risco para o adoecimento renal ou DRC já instalada bem como à sua família.

\section{REFERÊNCIAS}

ABRAHÃO, B. P. Análise de fatores de risco para DRC em usuários do SUS. Juiz de Fora: [s.n.], 2016.

ARAÚJO, L. F. S. et al. Diário de pesquisa e suas potencialidades na pesquisa qualitativa em saúde. Rev. Bras. Pesq. Saúde, Vitória, ES, v. 15, n. 3, p. 53-61, jul-set, 2013.

AVILA, A. C. et al. Aplicação do $5 \mathrm{~W} 2 \mathrm{H}$ para criação do manual interno de segurança do trabalho. Rev. Espacios, v. 37, n. 20, 2016.

BASTOS, M. G.; BREGMAN, R.; KIRSZTAJN, G. M. Doença renal crônica: frequente e grave, mas também prevenível e tratável. Rev. Assoc. Med. Bras., v. 56, n. 2, p. 248-253, set. 2017. Disponível em: http://www.scielo.br/pdf/ramb/ v56n2/a28v56n2 .pdf 9. Acesso em: 10 maio 2020.

BORBA, A. K. О. T. et al. Pesquisa e uso da informação em saúde: estruturação de projetos de intervenção em saúde. Recife: Una-SUS; Ufpe, 2015.

BORDENAVE, J. D.; PEREIRA, A. M. P. Estratégias de ensino-aprendizagem. 25. ed. Rio de Janeiro: Vozes, 2004.

BRASIL. Ministério da Saúde. Resolução no 466, de 12 de dezembro de 2012. Brasília, 2012. Disponível em: http://www. conselho.saude.gov.br/web_comissoes/conep/index.html. Acesso em: 4 jan. 2020.

BRASIL. Ministério da Saúde. Política Nacional de Promoção de saúde. 3. ed. Brasília: Ministério da Saúde, 2014. Disponívelem: http://bvsms.saude.gov.br/bvs/publicacoes/politica_nacional_promocao_saude_3ed.pdf. Acesso em: 30 ago. 2019. 
BRASIL. Ministério da Saúde. Estratégias para o cuidado da pessoa com doença crônica: diabetes mellitus. Cadernos de Atenção Básica, Brasília: MS, n. 36, 2013.

BRASIL. Ministério da Saúde, Gabinete do Ministro. Portaria no 2.436, de 21 de setembro de 2017. Aprova a Política Nacional de Atenção Básica, estabelecendo a revisão de diretrizes para a organização da Atenção Básica, no âmbito do Sistema Único de Saúde (SUS) [Internet]. Brasília: Ministério da Saúde, 2017.

CALDAS, M. L. et al. Doença renal crônica e atenção primária: desafios e perspectivas: revisão de literatura. Teresina: Conbracis, 2013.

COSTA, F. J. Mensuração e desenvolvimento de escalas: aplicações em administração. Rio de Janeiro: Ciência Moderna, 2011.

DIAS, A. C. A. Doença renal crônica e atenção primária desafios e perspectivas: revisão de literatura. Santa Luzia: [s.n.], 2014.

FASSBINDER, T. R. C. et al. Capacidade funcional e qualidade de vida de pacientes com doença renal crônica pré-dialítica e em hemodiálise - um estudo transversal. J Bras Nefrol, v. 37, n. 1, p. 47-54, 2015. Disponível em: https://www.scielo. br/pdf/jbn/v37n1/0101-2800-jbn-37-01-0047.pdf.

FERNANDES, L. C. L.; MACHADO, R. Z.; ANSCHAU, G. O. Gerência de serviços de saúde: competências desenvolvidas e dificuldades encontradas na atenção básica. Ciência \& Saúde Coletiva, v. 14 (supl.1), p. 541-1552, 2009.

HOSSAIN, M. M.; LADITKA, J. N. Using hospitalization for ambulatory care sensitive conditions to measure access to primary health care: an application of spatial structural equation modeling. Int J HealthGeogr, 8(1), p. 51, 2009.

IBGE. Instituto Brasileiro de Geografia e Estatística. Censo 2016. Disponível em: http://www.ibge.gov.br/censo2010/. Acesso em: 26 nov. 2019.

JACQUES, C. C.; MILANEZ, B.; MATTOS, R. de C. O. da C. Indicadores para Centros de Referência em Saúde do Trabalhador: proposição de um sistema de acompanhamento de serviços de saúde. Ciênc. Saúde Coletiva, v. 17, n. 2, p. 369-378, 2012. ISSN 1413-8123. Disponível em: https://www.scielo. $\mathrm{br} / \mathrm{scielo}$. php?pid=S1413-81232012000200011\&script $=\mathrm{s}-$ ci_abstract\&tlng=pt. Acesso em: 10 jul. 2020.

KLEIN, V.; BARIN, C. S. O arco de Maguerez como estruturador do ensino de química na Modalidade EJA. ENCONTRO DE PESQUISADORES DE EDUCAÇÃO A DISTÂNCIA. 2018, Santa Maria, RS, 2018.

LINO, D. P. Plano de intervenção para prevenir doença renal crônica em pacientes hipertensos do Centro de Saúde Vila Cemig, em Belo Horizonte - Minas Gerais. Belo Horizonte: [s.n.], 2016.

MALTA, D. C. et al. Probabilidade de morte prematura por doenças crônicas não transmissíveis, Brasil e regiões, projeções para 2025. Rev. Bras. Epidemiol., 2019 Disponível em: https://www.scielo.br/pdf/rbepid/v22/1980-5497-rbepid-22-e190030.pdf. Acesso em: 22 jul. 2020.
MELO, A. P. M.; MESQUITA, G. V.; MONTEIRO, C. F. S. Diagnóstico precoce da doença renal. R. Interd., v. 6, n. 1, p. 124-128, mar. 2013.

MENDES, E. V. O cuidado das condições crônicas na atenção primária à saúde: o imperativo da consolidação da estratégia da saúde da família. Brasília: Organização Pan-Americana da Saúde, 2012.

MENDES, E. V. O cuidado das condições crônicas na atenção primária à saúde. Rev. Bras. Promoç. Saúde, Fortaleza, 31(2), p. 1-3, abr./jun. 2018.

MENDES, R.; PEZZATO, L. M.; SACARDO, D. P. Pesquisa-intervenção em promoção da saúde: desafios metodológicos de pesquisar "com". Ciência \& Saúde Coletiva, São Paulo, 21(6), p. 1.737-1.745, 2016.

PEREIRA, A. C. et al. Associação entre fatores de risco clínicos e laboratoriais e progressão da doença renal crônica pré-dialítica. J. Bras. Nefrol., v. 34, n. 1, p. 68-75, 2013.

PRADO, M. T. et al. Arco de Charles Maguerez: Refletindo estratégias de metodologia ativa na formação de profissionais de saúde. Escola Anna Nery, v. 16, n. 1, p. 172-177, jan./mar. 2012.

REIS, et al. Tudo a temer: financiamento, relação público e privado e o futuro do SUS. Saúde Debate, Rio de Janeiro, v. 40, n. especial, p. 122-135, dez. 2016.

SANTOS, J. F. R. et al. Estratégias da atenção básica na doença renal crônica: a importância do diagnóstico precoce. São Paulo: [s.n.], 2017.

SERAPIONI, M. Pluralidade metodológica e interdisciplinaridade na pesquisa em serviços de saúde, 2012. Ciência \& Saúde Coletiva, 17, 3, 589-592. Disponível em: https://ces. uc.pt/myces/UserFiles/livros/1097_Pluralidade\%20metodol\%F3gica\%20e\%20interdisciplinaridade\%20C\%26SC.pdf. Acesso em: 20 jul. 2020.

SILVA, L. B. Qualidade do cuidado à pessoa idosa com diabetes e/ou hipertensão atendida na Atenção Primária à Saúde. Belo Horizonte: [s.n.], 2018. Disponível em: http://hdl. handle.net/1843/ENFC-B6HRC4. Acesso em: dez. 2019.

SOBRAL. Secretaria de Saúde Municipal de Sobral. Reterritorialização do Bairro Sumaré. Sobral, 2016.

VIANA, D. M. S. et al. A educação permanente em saúde na perspectiva do enfermeiro na estratégia de saúde da família. 2015. Disponível em: http://seer.ufsj.edu.br/index.php/ recom/article/view/470. Acesso em: 20 dez. 2019.

VILLARDI, M. L.; CYRINO, E. G.; BERBEL, N. A. N. A metodologia da problematização no ensino em saúde: suas etapas e possibilidades. In: VILLARDI, M. L.; CYRINO, E. G.; BERBEL, N. A. N. A problematização em educação em saúde: percepções dos professores tutores e alunos [on-line]. São Paulo: Cultura Acadêmica; Editora Unesp, 2015. 